\title{
TIME-OPTIMAL HEURISTIC ALGORITHMS FOR FINDING CLOSEST-PAIR OF POINTS IN 2D AND 3D
}

\author{
Mashilamani Sambasivam \\ (formerly) Department of Computer Science, Texas A\&M University, USA \\ kandan1976 doutlook.com
}

\begin{abstract}
Given a set of $n$ points in $2 D$ or $3 D$, the closest-pair problem is to find the pair of points which are closest to each other. In this paper, we give a new $O(n \log n)$ time algorithm for both $2 D$ and $3 D$ domains. In order to prove correctness of our heuristic empirically, we also provide java implementations of the algorithms. We verified the correctness of this heuristic by verifying the answer it produced with the answer provided by the brute force algorithm, through 600 trial runs, with different number of points. We also give empirical results of time taken by running our implementation with different number of points in both $2 D$ and $3 D$.
\end{abstract}

\section{KEYWORDS}

Closest-pair, Algorithm, Heuristic, Time-Optimal, Computational Geometry, 2D, 3D

\section{INTRODUCTION}

The closest-pair solution has many applications in real-life. It forms a main step in many problem-solving procedures. These include applications in air/land/water traffic-control systems. A traffic control system can use the solution in order to avoid collisions between vehicles. The algorithm has applications in detecting collisions after they happen. There are also applications in self-navigating vehicles. The solution also has applications in bodies which must always keep close to particular other bodies. The problem also has applications in imaging technologies, pattern recognition, CAD, VLSI.

\section{PREVIOUS WORK}

The most popular algorithm in 2D appears in the book by Cormen et al[1] and is due to Preparata and Shamos[2]. The algorithm divides the problem spatially and uses a divide-and conquer method. Following this algorithm, many similar divide-and-conquer algorithms have been devised for 3D by dividing the points spatially by a plane.[3][4][5] contain a good survey of computational geometry algorithms. Our algorithm differs from previous algorithms in that it is much simpler and therefore much easier to implement practically. The previous best algorithms for $2 \mathrm{D}$ have a time bound of $\mathrm{O}(\mathrm{n} \log \mathrm{n})$ similar to our $2 \mathrm{D}$ algorithm. However, I am unable to Natarajan Meghanathan et al. (Eds) : ACSIT, FCST, ITCA, CoNeCo - 2015 pp. 07-13, 2014. ( C CS \& IT-CSCP 2015

DOI : $10.5121 /$ csit.2015.51202 
establish the best time bound achieved by previous algorithms for 3D. I think the best time bound achieved by previous algorithms for $3 \mathrm{D}$ is $\mathrm{O}\left(\mathrm{n}^{*} \log ^{2} \mathrm{n}\right)$.

\section{OUR ALGORITHMS}

We present the 2D and 3D algorithms separately for clarity.

\subsection{Algorithm for 2D}

Algorithm 2D-ClosestPair( )

Given: $\mathrm{n}-$ number of points, $\mathrm{p}[1 . . \mathrm{n}]-$ points array

Data structures used by algorithm:

$\mathrm{d} 1[1 . . n], \mathrm{d} 2[1 . . \mathrm{n}], \mathrm{d} 3[1 . . \mathrm{n}], \mathrm{d} 4[1 . . \mathrm{n}]$ - distance arrays

$\operatorname{sum}[1 . . n]-$ sum array

index $[1 . . n]-$ index array

1. a. Find point $\mathrm{p} 1$ such that its $\mathrm{x}$ coordinate is lower or equal to any other point in the array of points $\mathrm{p}$.

b. Find point $\mathrm{p} 2$ such that its $\mathrm{x}$ coordinate is higher or equal to any other point in the array of points $p$.

c. Find point p3 such that its y coordinate is lower or equal to any other point in the array of points $\mathrm{p}$.

d. Find point p4 such that its y coordinate is higher or equal to any other point in the array of points $p$.

2. a. Find distance of each point in the $\mathrm{p}$ array from $\mathrm{p} 1$ and put its square in the $\mathrm{d} 1$ array.

For $\mathrm{i}=1 . . \mathrm{n}, \quad \mathrm{d} 1[\mathrm{i}]=(\text { distance between } \mathrm{p} 1 \text { and } \mathrm{p}[\mathrm{i}])^{2}$

b. Find distance of each point in the p array from $\mathrm{p} 2$ and put its square in the $\mathrm{d} 2$ array.

For $\mathrm{i}=1 . . \mathrm{n}, \mathrm{d} 2[\mathrm{i}]=(\text { distance between } \mathrm{p} 2 \text { and } \mathrm{p}[\mathrm{i}])^{2}$

c. Find distance of each point in the $\mathrm{p}$ array from $\mathrm{p} 3$ and put its square in the $\mathrm{d} 3$ array.

For $\mathrm{i}=1 . . \mathrm{n}, \mathrm{d} 3[\mathrm{i}]=(\text { distance between } \mathrm{p} 3 \text { and } \mathrm{p}[\mathrm{i}])^{2}$

$\mathrm{d}$. Find distance of each point in the $\mathrm{p}$ array from $\mathrm{p} 4$ and put its square in the $\mathrm{d} 4$ array.

For $\mathrm{i}=1 . . \mathrm{n}, \mathrm{d} 4[\mathrm{i}]=(\text { distance between } \mathrm{p} 4 \text { and } \mathrm{p}[\mathrm{i}])^{2}$ 
3. Calculate the sum array using the following formula:

For $\mathrm{i}=1 . . \mathrm{n}, \quad \operatorname{sum}[\mathrm{i}]=11 * \mathrm{~d} 1[\mathrm{i}]+101 * \mathrm{~d} 2[\mathrm{i}]+1009 * \mathrm{~d} 3[\mathrm{i}]+10007 * \mathrm{~d} 4[\mathrm{i}]$

4. Initialise the index array to contain the indexes.

For $\mathrm{i}=1 . . \mathrm{n}, \quad$ index $[\mathrm{i}]=\mathrm{i}$

5. Mergesort the sum array. While mergesorting, if you exchange any 2 indices $i$ and $j$ of sum array, be sure to exchange the corresponding entries $i$ and $j$ of index array.

6. For $\mathrm{i}=1 . .(\mathrm{n}-1)$, Compare each point $\mathrm{p}[\mathrm{index}[\mathrm{i}]]$ to the 10 next points (if they exist).

ie. $p[$ index $[i+1]], p[$ index $[i+2]] . . p[i n d e x[i+10]]$

If the 2 points being compared is the closest pair found so far, then store the 2 points.

7. Output the closest pair of points found.

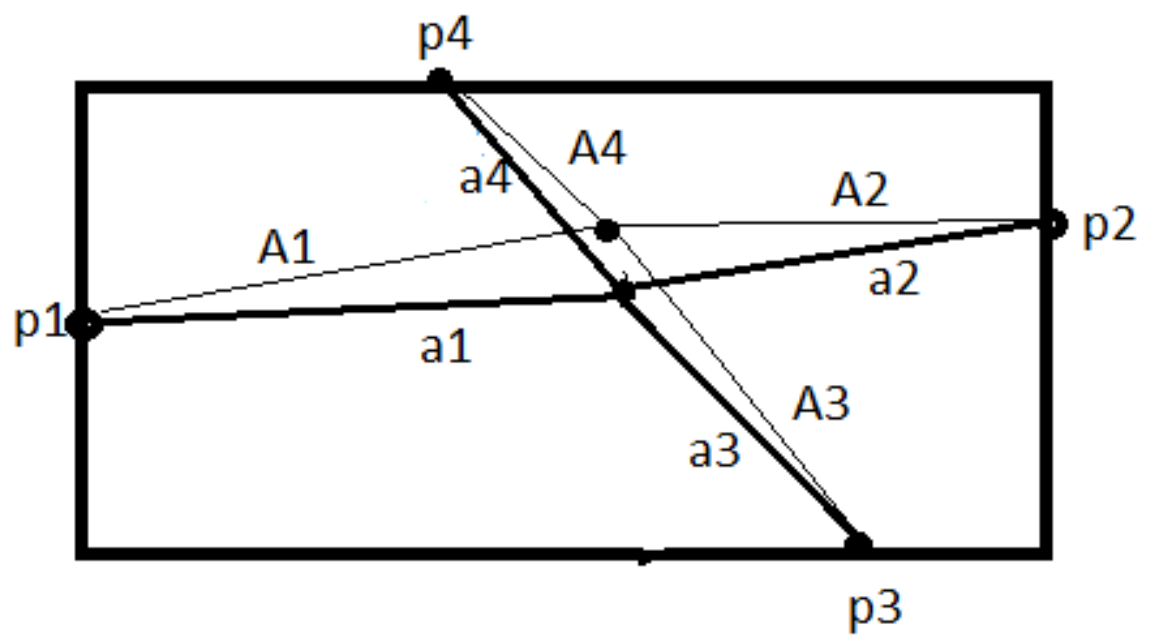

Figure 1. Closest Pair

Assume p1, p2, p3, p4, seen in the Figure 1 above, are the extreme points found in step 1 of our algorithm. Then the basic idea of our algorithm is that the closest pair of points (the 2 points inside the rectangle) should be almost equidistant from each of the 4 points (see Figure 1 above). That is, a1 should be near A1 numerically, and a2 should be near A2 numerically, and a3 should be near A3 numerically, and a4 should be near A4 numerically.

So what our algorithm does is that it calculates the distance of each point from the 4 extreme points and puts its square in the corresponding $\mathrm{d}$ array. We wish to find $(\mathrm{d} 1, \mathrm{~d} 2, \mathrm{~d} 3, \mathrm{~d} 4)$ of a point $\mathrm{x}$ such that it almost equals $(\mathrm{d} 1, \mathrm{~d} 2, \mathrm{~d} 3, \mathrm{~d} 4)$ of a point $\mathrm{y}$. The closer the match of the d's, the closer the points are in the $2 \mathrm{D}$ plane. 
So what we do to find the closest match of $(\mathrm{d} 1, \mathrm{~d} 2, \mathrm{~d} 3, \mathrm{~d} 4)$ among all points in the $\mathrm{d}$ array, is that we multiply each by a prime number and add them to get the sum array. The closer the $(\mathrm{d} 1, \mathrm{~d} 2$, $\mathrm{d} 3, \mathrm{~d} 4)$ of point $\mathrm{x}$ is to $(\mathrm{d} 1, \mathrm{~d} 2, \mathrm{~d} 3, \mathrm{~d} 4)$ of point $\mathrm{y}$, the closer will be the sum numerically. Multiplying by prime numbers gives us a unique signature of each point in the sum array. Note that the prime numbers are all different from each other.

So then, we let the index array carry the index of the point corresponding to the sum array. We then mergesort the sum array, taking care to exchange corresponding entries of index array when we exchange 2 elements of the sum array.

Now, we have the sorted sum array, and the points they represent are in the index array. Now, all we have to do is compare each point in the index array with 10 points that follow it. If the distance between 2 points being compared is the closest pair we have so far, it get stored. The closest pair of points is then output.

\subsection{Algorithm for 3D}

\section{Algorithm 3D-ClosestPair( )}

Given: $\mathrm{n}$ - number of points, $\mathrm{p}[1 . . \mathrm{n}]-$ points array

Data structures used by algorithm:

d1[1..n] , d2[1..n], d3[1..n], d4[1..n], d5[1..n], d6[1..n] - distance arrays

$\operatorname{sum}[1 . . n]-$ sum array, $\quad$ index[1..n] - index array

1. a. Find point $\mathrm{p} 1$ such that its $\mathrm{x}$ coordinate is lower or equal to any other point in the array of points $\mathrm{p}$.

b. Find point $\mathrm{p} 2$ such that its $\mathrm{x}$ coordinate is higher or equal to any other point in the array of points $p$.

c. Find point $\mathrm{p} 3$ such that its y coordinate is lower or equal to any other point in the array of points $\mathrm{p}$.

d. Find point $\mathrm{p} 4$ such that its y coordinate is higher or equal to any other point in the array of points $p$.

e. Find point $\mathrm{p} 5$ such that its $\mathrm{z}$ coordinate is lower or equal to any other point in the array of points $\mathrm{p}$.

f. Find point p6 such that its $\mathrm{z}$ coordinate is higher or equal to any other point in the array of points $\mathrm{p}$.

2. a. Find distance of each point in $\mathrm{p}$ array from $\mathrm{p} 1$ and put its square in the $\mathrm{d} 1$ array.

For $\mathrm{i}=1 . . \mathrm{n}, \quad \mathrm{d} 1[\mathrm{i}]=(\text { distance between } \mathrm{p}[\mathrm{i}] \text { and } \mathrm{p} 1)^{2}$ 
b. Find distance of each point in $\mathrm{p}$ array from $\mathrm{p} 2$ and put its square in the $\mathrm{d} 2$ array For $\mathrm{i}=1 . . \mathrm{n}, \mathrm{d} 2[\mathrm{i}]=(\text { distance between } \mathrm{p}[\mathrm{i}] \text { and } \mathrm{p} 2)^{2}$

c. Find distance of each point in $\mathrm{p}$ array from $\mathrm{p} 3$ and put its square in the $\mathrm{d} 3$ array. For $\mathrm{i}=1 . . \mathrm{n}, \mathrm{d} 3[\mathrm{i}]=(\text { distance between } \mathrm{p}[\mathrm{i}] \text { and } \mathrm{p} 3)^{2}$

d. Find distance of each point in $\mathrm{p}$ array from $\mathrm{p} 4$ and put its square in the $\mathrm{d} 4$ array. For $\mathrm{i}=1 . . \mathrm{n}, \mathrm{d} 4[\mathrm{i}]=(\text { distance between } \mathrm{p}[\mathrm{i}] \text { and } \mathrm{p} 4)^{2}$

e. Find distance of each point in $\mathrm{p}$ array from $\mathrm{p} 5$ and put its square in the $\mathrm{d} 5$ array. For $\mathrm{i}=1 . . \mathrm{n}, \mathrm{d} 5[\mathrm{i}]=(\text { distance between } \mathrm{p}[\mathrm{i}] \text { and } \mathrm{p} 5)^{2}$

f. Find distance of each point in p array from p6 and put its square in the d6 array. For $\mathrm{i}=1 . . \mathrm{n}, \mathrm{d} 6[\mathrm{i}]=(\text { distance between } \mathrm{p}[\mathrm{i}] \text { and } \mathrm{p} 6)^{2}$

3. Calculate the sum array using the following formula:

For $\mathrm{i}=1 . . \mathrm{n}$,

$$
\operatorname{sum}[\mathrm{i}]=11 * \mathrm{~d} 1[\mathrm{i}]+101 * \mathrm{~d} 2[\mathrm{i}]+547 * \mathrm{~d} 3[\mathrm{i}]+1009 * \mathrm{~d} 4[\mathrm{i}]+5501 * \mathrm{~d} 5[\mathrm{i}]+10007 * \mathrm{~d} 6[\mathrm{i}]
$$

4. Initialise the index array to contain the indexes.

For $\mathrm{i}=1 . . \mathrm{n}, \quad$ index $[\mathrm{i}]=\mathrm{i}$

5. Mergesort the sum array. While mergesorting, if you exchange any 2 indices $i$ and $j$ of sum array, be sure to exchange the corresponding entries $i$ and $j$ of index array.

6. For $\mathrm{i}=1 . .(\mathrm{n}-1)$, Compare each point $\mathrm{p}[\mathrm{index}[\mathrm{i}]]$ to the 100 next points (if they exist) ie. $p[$ index $[i+1]], p[i n d e x[i+2]] . . p[i n d e x[i+100]]$

if the 2 points being compared is the closest pair found so far, then store the 2 points.

7. Output the closest pair of points found.

Assume p1, p2, p3, p4, p5, p6 are the extreme points found in step 1 of our 3D algorithm. Then the basic idea of our algorithm is that the closest pair of points should be almost equidistant from each of the 6 points.

So what our algorithm does is that it calculates the distance of each point from the 6 extreme points and puts its square in the corresponding $\mathrm{d}$ array. We wish to find $(\mathrm{d} 1, \mathrm{~d} 2, \mathrm{~d} 3, \mathrm{~d} 4, \mathrm{~d} 5, \mathrm{~d} 6)$ of a point $\mathrm{x}$ such that it almost equals $(\mathrm{d} 1, \mathrm{~d} 2, \mathrm{~d} 3, \mathrm{~d} 4, \mathrm{~d} 5, \mathrm{~d} 6)$ of a point $\mathrm{y}$. The closer the match of the d's, the closer the points are in 3D.

So what we do to find the closest match of $(\mathrm{d} 1, \mathrm{~d} 2, \mathrm{~d} 3, \mathrm{~d} 4, \mathrm{~d} 5, \mathrm{~d} 6)$ among all points in the d array, is that we multiply each by a prime number and add them to get the sum array. The closer the $(\mathrm{d} 1, \mathrm{~d} 2, \mathrm{~d} 3, \mathrm{~d} 4, \mathrm{~d} 5, \mathrm{~d} 6)$ of point $\mathrm{x}$ is to $(\mathrm{d} 1, \mathrm{~d} 2, \mathrm{~d} 3, \mathrm{~d} 4, \mathrm{~d} 5, \mathrm{~d} 6)$ of point $\mathrm{y}$, the closer will be the 
sum numerically. Multiplying by prime numbers gives us a unique signature of each point in the sum array. Note that the prime numbers are all different from each other.

So then, we let the index array carry the index of the point corresponding to the sum array. We then mergesort the sum array, taking care to exchange corresponding entries in the index array when we exchange 2 elements of the sum array.

Now, we have the sorted sum array, and the points they represent are in the index array. Now, all we have to do is compare each point in the index array with 100 points that follow it. If the distance between 2 points being compared is the closest pair we have so far, it get stored.The closest pair of points is then output.

\subsection{Correctness of our Heuristic Algorithm}

We implemented our algorithms in 2D and 3D in java. The programs can be downloaded from the private url: https://drive.google.com/file/d/0B2MLVfnv5msBVlBnWEthcjRkM00/view?

usp=sharing. We ran 600 trial runs with number of points ranging from 1 hundred to 10 million. We verified the answer we got with the answer got from the brute force algorithm of finding the closest pair. Our program got it right $100 \%$ of time.

The correctness of our heuristic is also intuitive-that the closest-pair of points will be almost equidistant from each of the extreme points found. Also, multiplying by a prime is intuitive in that it gives us a unique signature of each point in the sum array.

\subsection{Running Time of our algorithm}

Each of the steps in our algorithm takes $\mathrm{O}(\mathrm{n})$ time, except the mergesort step5. Mergesort step takes $\mathrm{O}(\mathrm{n} \log \mathrm{n})$ time. Note that the $6^{\text {th }}$ step takes $\mathrm{O}(10 \mathrm{n})$ for $2 \mathrm{D}$ algorithm and $\mathrm{O}(100 \mathrm{n})$ for $3 \mathrm{D}$ algorithm, which is essentially $\mathrm{O}(\mathrm{n})$ time. So the total time taken by our algorithm is $\mathrm{O}(\mathrm{n} \log n)$. The following tables gives the running time of our algorithm with varying number of points. It compares the running time against the running time of a brute force $\mathrm{O}\left(\mathrm{n}^{2}\right)$ algorithm. Each entry in the table (except brute-force algorithm entries for 1 million and 10 million points) is the average time of running the algorithm over 50 trial runs. The trials were run on a single-processor with base frequency of $1.6 \mathrm{GHz}$.

Table 1. Running time of our 2D algorithm and brute-force algorithm

\begin{tabular}{|l|l|l|}
\hline Number of Points & Our 2D algorithm time & Brute force algorithm time \\
\hline 1000 & 17 millisecs & 47 millisecs \\
\hline 10000 & 70 millisecs & 1200 millisecs \\
\hline 100000 & 330 millisecs & 99 secs \\
\hline 1 million & 1.7 secs & $>12$ hours \\
\hline 10 million & 15.5 secs & $>>12$ hours \\
\hline
\end{tabular}


Table 2. Running time of our 3D algorithm and brute-force algorithm

\begin{tabular}{|l|l|l|}
\hline Number of Points & Our 3D algorithm time & Brute force algorithm time \\
\hline 1000 & 54 millisecs & 49 millisecs \\
\hline 10000 & 165 millisecs & 1700 millisecs \\
\hline 100000 & 731 millisecs & 139 secs \\
\hline 1 million & 5.2 secs & $>12$ hours \\
\hline 10 million & 47 secs & $>>12$ hours \\
\hline
\end{tabular}

\section{CONCLUSions}

We found our heuristic algorithm gives the right answer $100 \%$ of time. Since the algorithm's correctness cannot be proved mathematically, it is still a heuristic. However, we have proved our algorithm's correctness empirically. Our algorithm is also time-optimal in that both the algorithms for $2 \mathrm{D}$ and $3 \mathrm{D}$ run in $\mathrm{O}(\mathrm{n} \log \mathrm{n})$ time. We verified empirically that our algorithm is time optimal.

Future work in finding closest pair of points can include finding the pair with multicores/multiprocessors, which are becoming more common day to day.

\section{ACKNOWLEDGEMENTS}

The author would like to thank Perumal. S, Sambasivam. K and Shankari. S for their support.

\section{REFERENCES}

[1] Thomas H. Cormen, Charles E. Leiserson, Ronald L. Rivest \& Clifford Stein (2009) Introduction to Algorithms, PHI Learning, Eastern Economy Edition.

[2] Franco P. Preparata \& Michael Ian Shamos (1985) Computational Geometry: An Introduction, Springer.

[3] Herbert Edelsbrunner (1987) Algorithms in Combinatorial Geometry, Vol. 10 of EATCS Monographs on Theoretical Computer Science, Springer.

[4] Joseph O'Rourke (1998) Computational Geometry in C, Cambridge University Press.

[5] Mark de Berg, Otfried Cheong, Marc van Kreveld \& Mark Overmars (2011) Computational Geometry: Algorithms and Applications, Springer.

\section{AUTHORS}

Mashilamani. S holds a Masters degree in Computer Science from Texas A\&M University, College Station, USA and a Bachelors in Computer Science and Eng. from Madras University.

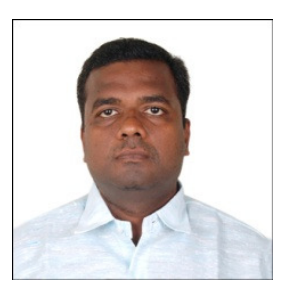

